POS PROCEEDINGS

\title{
Ultra Long-Lived Particles with MATHUSLA
}

\author{
Cristiano Alpigiani ${ }^{*+}$ \\ University of Washington, Seattle \\ E-mail: Cristiano.Alpigiani@cern.ch
}

\begin{abstract}
Many extensions of the Standard Model (SM) include particles that are neutral, weakly coupled, and long-lived that can decay to final states containing several hadronic jets. Long-lived particles (LLPs) can be detected as displaced decays from the interaction point, or missing energy if they escape. ATLAS and CMS have performed searches at the LHC and significant exclusion limits have been set in recent years. However, the current searches performed at colliders have limitations. An LLP does not interact with the detector and it is only visible once it decays. Unfortunately, no existing or proposed search strategy will be able to observe the decay of non-hadronic electrically neutral LLPs with masses above $\sim \mathrm{GeV}$ and lifetimes near the limit set by Big Bang Nucleosynthesis $\left(c \tau \sim 10^{7}-10^{8} \mathrm{~m}\right)$. Therefore, ultra-long-lived particles (ULLPs) produced at the LHC will escape the main detector with extremely high probability. We describe the concept of the MATHUSLA surface detector (MAssive Timing Hodoscope for Ultra Stable neutraL pArticles), which can be implemented with existing technology and in time for the high luminosity LHC upgrade to find such ultra-long-lived particles, whether produced in exotic Higgs decays or more general production modes. The MATHUSLA detector will consist of resistive plate chambers (RPC) and scintillators with a total sensitive area of $200 \times 200 \mathrm{~m}$ square. It will be installed on the surface, close to the ATLAS or CMS detectors. A small-scale test detector $\left(\sim 6 \mathrm{~m}^{2}\right)$ has been installed on the surface above ATLAS in autumn 2017. It consists of three layers of RPCs used for timing/tracking and two layers of scintillators for timing measurements. It has been placed above the ATLAS interaction point to estimate cosmic backgrounds and proton-proton backgrounds coming from ATLAS during nominal LHC operations.
\end{abstract}

An Alpine LHC Physics Summit (ALPS2018)

15-20 April, 2018

Obergurgl, Austria

\footnotetext{
* Speaker.

${ }^{\dagger}$ On behalf of the MATHUSLA Collaboration.
} 


\section{The hidden sector}

The discovery of the Higgs boson at the LHC completed the SM and focused attention on the many central features of our universe that the SM does not address: dark matter (DM), neutrino mass, particle-antiparticle asymmetry (baryogenesis), hierarchy problem (naturalness). Many Beyond the Standard Model (BSM) theoretical constructs proposed in the past few years that address these phenomena contain LLPs with macroscopic decay lengths limited only by Big Bang Nucleosynthesis (BBN) of about $c \tau \lesssim 10^{7}-10^{8} \mathrm{~m}$. Examples include, Supersymmetric (SUSY) models such as mini split SUSY [1], gauge mediation [2], RPV SUSY [3] and Stealth SUSY [4]. Models addressing the hierarchy problem such as Neutral Naturalness [5] and Hidden Valleys [6, 7], and models addressing dark matter [8].

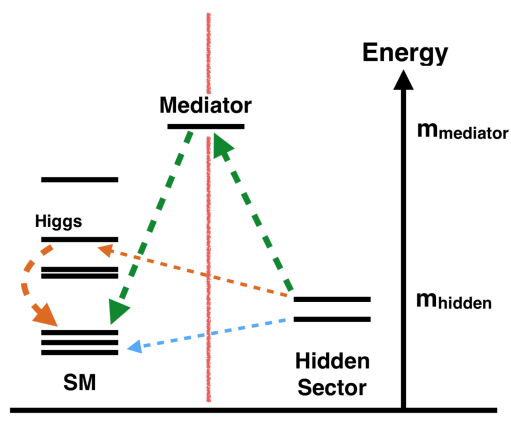

(a)

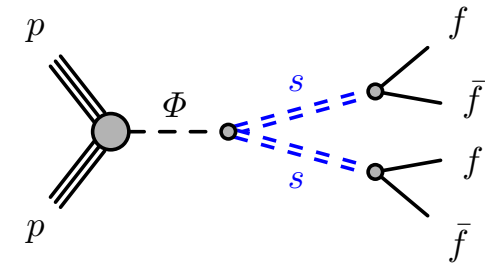

(b)

Figure 1: Schematic of the coupling between the SM and a hidden sector (a) and Feynman diagram for the Higgs decaying into displaced hadronic jets (b).

LLPs occur naturally in coupling to a hidden sector via small portal couplings. For example scalar (Higgs) and vector $\left(\gamma_{d}, Z\right)$ portals $[6,7]$ where the Higgs boson mixes with a hidden sector scalar resulting in a hidden sector Higgs that decays to a pair of hidden sector scalars that carry no SM charge (shown schematically in Figure 1). If the hidden sector scalars are weakly coupled, they will have a long-lifetime and decay far from the IP ( $p p$ interaction point) to a fermion anti-fermion pair (quark anti-quark pairs). The detector signature is a hadronic jet displaced from the IP and no track connecting it to the IP. Similarly the kinetic mixing of a $\gamma_{d}$ results in dark photons that decay to lepton pairs giving rise to a pair of displaced lepton vertices in the detector. The hidden sector particles have no SM quantum numbers and once produced travel through the detector unabated. Once they decay to SM particles a detector signal is observed. The salient signature is a displaced vertex with no tracks connecting to the IP.

Searches for LLPs in the LHC detectors have set significant $c \tau$ exclusion limits from a few centimetres to tens of meters[9, 10]. The large backgrounds from various SM processes (such as QCD jets) limit the $c \tau$ reach of the LHC detectors. The proposed MATHUSLA detector [11], which is a dedicated large volume, air filled detector located on the surface above and somewhat displaced from the ATLAS or CMS IPs, will be sensitive to lifetimes of $10^{7} \mathrm{~m}$ with the expected integrated luminosity of High Luminosity LHC (HL-LHC). 


\section{The MATHUSLA detector}

MATHUSLA [11, 12] is a proposed dedicated detector to observe Ultra Long-lived Particles (ULLPs) produced in $p p$ collisions that, because of their long lifetimes, decay to charged SM particles far from the main detector. The HL-LHC will produce $N_{h} \approx 1.5 \times 10^{8} \mathrm{Higgs}$ bosons.

When producing ULLPs in exotic Higgs decays, the number of observed ULLP decays is roughly:

$$
N_{\mathrm{obs}} \sim N_{h} \cdot \operatorname{Br}(h \rightarrow \mathrm{ULLP} \rightarrow \mathrm{SM}) \cdot \varepsilon_{\text {geometric }} \cdot \frac{L}{b c \tau},
$$

where $L$ is the linear size of the detector along the ULLP's direction of travel, $\varepsilon_{\text {geometric }}$ is the chance that the ULLP will pass through the detector (i.e. geometric coverage), and $b$ is the Lorentz boost $|\vec{p}| / m$ of the produced ULLP. Since the Higgs boson is dominantly produced on threshold, if it decays to $n$ ULLPs with mass $m_{X}$, their characteristic boost will be

$$
b \sim \frac{m_{h}}{n m_{X}},
$$

so typically $b \lesssim 3$ for $n=2$ and $m_{X} \gtrsim 20 \mathrm{GeV}^{1}$. We focus on $n=2$ which suffices to discuss detector requirements. Assuming the ULLP decays exclusively to the $\mathrm{SM}^{2}$, observation of a few ULLP decays with a lifetime of $c \tau \sim 10^{7} \mathrm{~m}$ requires

$$
L \sim(20 \mathrm{~m})\left(\frac{b}{3}\right)\left(\frac{0.1}{\varepsilon_{\text {geometric }}}\right) \frac{0.3}{\mathrm{Br}(h \rightarrow \mathrm{ULLP})} .
$$

ULLPs near the BBN lifetime bound arising from exotic Higgs decays near current limits [13] could be discovered if the detector had a linear size of $\sim 20 \mathrm{~m}$ in the direction of travel and $\sim 10 \%$ geometric coverage and did not have $p p$ collision backgrounds.

Placing a sufficiently large area detector on the surface, as shown in Figure 2, above the ATLAS or CMS collision points ( 80 to $100 \mathrm{~m}$ below) gives sufficient acceptance to reach the BBN lifetime limit [11]. The 80 to 100 meters of rock removes most backgrounds associated with $p p$ collisions, but a large background of cosmic muons and backgrounds from high energy muons and neutrinos coming from the IP must be rejected. The proposed detector concept, shown in Figure 2 , is basically a large box surrounded by a layer of scintillators, a robust tracking system (about 5 layers of tracking chambers) and a few meter air decay volume. Three simplified geometries for the MATHUSLA decay volume are currently under studies. To a reasonable approximation, sensitivity to LLP production rate (long lifetime) scales inversely (linearly) with detector area, assuming fixed height. As long as the detector starts $\lesssim 100 \mathrm{~m}$ horizontally displaced from the IP on the surface, the sensitivity is not greatly dependent on the precise position of the detector. MATHUSLA200 has a $200 \mathrm{~m} \times 200 \mathrm{~m} \times 20 \mathrm{~m}$ decay volume. This is the geometry proposed in [11] and studied in most previous works, including the physics case white paper [12]. Significantly, this large size would allow MATHUSLA to probe LLP lifetimes close to the BBN limit $c \tau \lesssim 10^{7} \mathrm{~m}$ for LLPs produced in exotic Higgs decays. It could be implemented if sufficient land was made available, but the default benchmark currently under consideration is MATHUSLA100, which is smaller by a factor

\footnotetext{
${ }^{1} n>2$ typically implies higher-dimensional operators, cascade decays in the hidden sector, or a hidden confining gauge group that produces a shower.

${ }^{2}$ Naively, a coincidence of small widths is required for a ULLP to decay to both SM and hidden sector particles.
} 

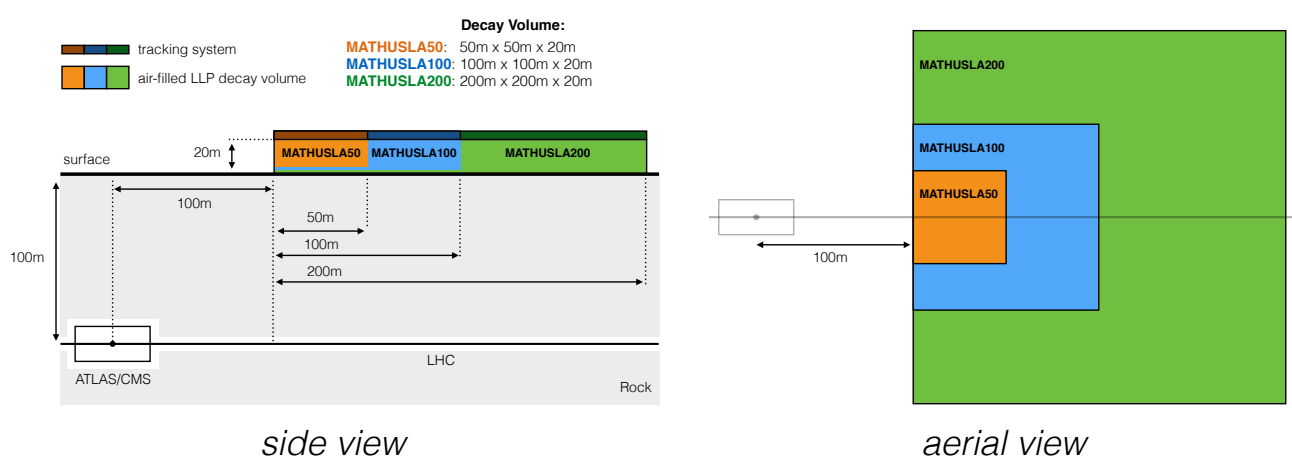

Figure 2: Possible simplified MATHUSLA geometries with different decay volumes. MATHUSLA100 is the current default benchmark, since it is approximately the right size for an available experimental site near CMS. MATHUSLA200 is the geometry proposed in [11] and studied in [12]. It can probe the BBN lifetime limit for LLPs produced in exotic Higgs decays, and could be implemented if sufficient area was made available. The smaller MATHUSLA50 is considered alongside the other two geometries to demonstrate the scalability of the design.

of 4 with a $100 \mathrm{~m} \times 100 \mathrm{~m} \times 20 \mathrm{~m}$ decay volume. Sensitivity projections for various geometries assuming 4 events decaying in MATHUSLA are shown in Figure 3.

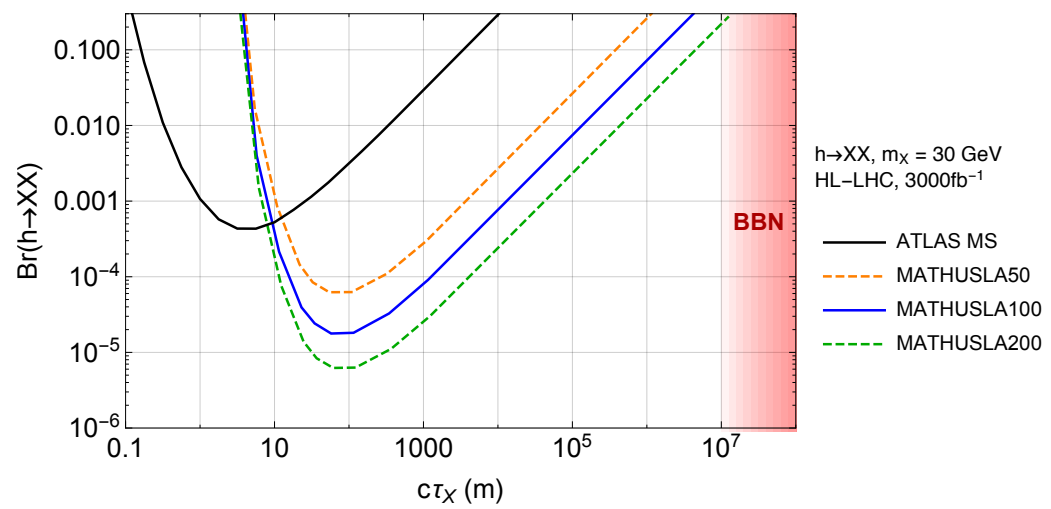

Figure 3: Comparison of reach for $30 \mathrm{GeV}$ LLPs produced in exotic Higgs decays and decaying hadronically, for various MATHUSLA geometries (curves correspond to 4 LLPs decaying in the detector volume) and the LHC main detector exclusion projection using a single-DV search in the ATLAS Muon System [14].

Figure 4 shows one possible instrumentation of MATHUSLA assuming the geometry in Figure 2. The air-filled-decay volume is surrounded by a layer of plastic scintillators (top, bottom and sides) with a robust, highly redundant, multilayer tracker located at the top. The tracking system allows to reconstruct the trajectories of charged particles and provides timing information. One wellestablished candidate technology for this large area tracker are Resistive Plate Chambers (RPCs) that have $\mathrm{cm}$ spatial and $\mathrm{ns}$ timing resolution.

More in detail, Figure 4 (a) shows two possible displaced vertex signals from LLP decays, while potential backgrounds are shown symbolically in Figure 4 (b). They include a cosmic ray 


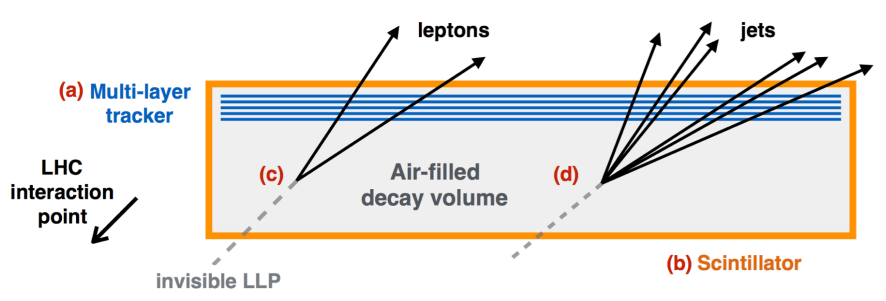

(a)

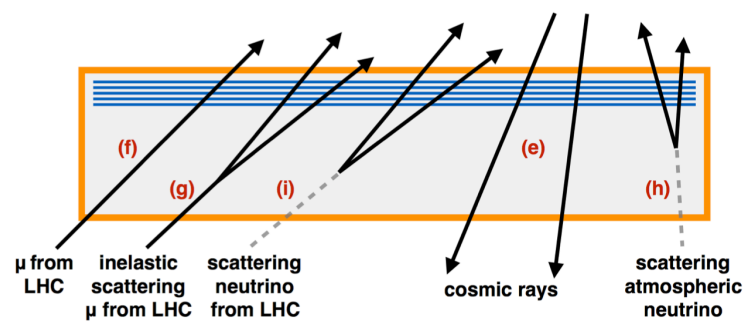

(b)

Figure 4: Schematic of a possible design for MATHUSLA, with a robust multi-layer tracker at the top and a segmented scintillator veto surrounding the entire detector. Also shown are two possible displaced vertex signals from LLP decays (a) and the five most important backgrounds (b). Black arrows indicate charged particles and their direction of travel.

muon rate of about $10^{6} \mathrm{~Hz}$. The very good time resolution of the scintillators (around $1.5 \mathrm{~ns}$ or better) and the good space and time resolution of the RPCs allow a powerful background rejection. The expected rate of LHC collision muons is about $10 \mathrm{~Hz}$ and are rejected by timing and entrance hit position. Upward going atmospheric neutrinos are estimated to be of order 10 to 100 per year and most can be rejected using time of flight. In addition, such non-collision backgrounds can be measured when there are no LHC collisions. Neutrinos from LHC collisions are estimated to a few events during the entire HL-LHC data taking period. This appears to be a subdominant background.

Efforts are underway to develop simulations of the backgrounds expected in the MATHUSLA detector. For muons and neutrinos traveling upwards, the idea is to create a "gun" that shoots particles into MATHUSLA, while for cosmic muons the plan is to use the standard cosmic ray simulations (CORSIKA). Nevertheless, the simulations need to be validated (and tuned) using real data.

\section{The MATHUSLA test module}

A test stand, with a detector layout similar to the one envisioned for the MATHUSLA detector, has been assembled at CERN. It was installed in the surface area above the ATLAS interaction point at LHC Point 1 in November 2017 and it has been taking data with different beam conditions. The main purpose of the test stand is to provide empirical information on potential backgrounds coming from the LHC as well as from cosmic rays. The MATHUSLA detector has been conceived to be a background-free detector. This hypothesis can be studied making use of this test stand by taking data with different LHC beams conditions. On one hand, data taken with no beams running in the LHC will provide a good measurement of the background from cosmic rays as well 
as good information about their trigger and identification. On the other hand, data collected with beams running in the LHC can give an estimate of the background expected from LHC collisions. A precise measure of the charged particle flux in the test stand will provide the veto efficiency requirement for the main detector. The goal is to achieve a timing resolution sufficient to guarantee that no cosmic particles can fake charged particle coming from LHC. All the tests that will be performed until the end of LHC Run 2 will be fundamental to understand the cosmic ray rate and to extrapolate the LHC-related background rate from the test stand to MATHUSLA. All this information can be used to optimize the final design of the main detector.

Following the concept of the main detector, the test stand is composed of two external layers of scintillators (one on the upper part of the detector, one on the lower part) with six layers of RPCs between them. Figures 5a and 5a show respectively the basic design of the test module and a picture of the final assembled structure in ATLAS SX1 building at CERN. The overall structure is $\sim 6.5 \mathrm{~m}$ tall, with an base of $\sim 2.5 \times 2.5 \mathrm{~m}^{2}$.

Top and bottom scintillator layers are coming from Tevatron $\mathrm{D} \emptyset$ forward muon trigger scintillators [18], while the RPCs (provided by University of Tor Vergata, Rome) are coming from the cosmic ray experiment Argo-YBJ [19] at the YangBaJing Laboratory in Tibet (4300 m a.s.l.).

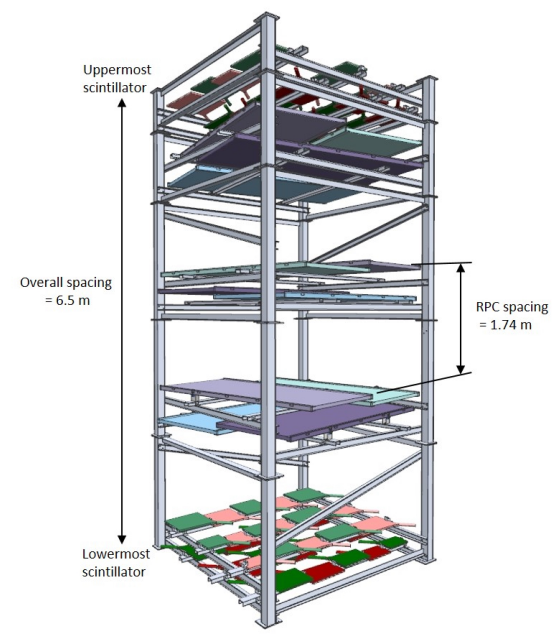

(a)

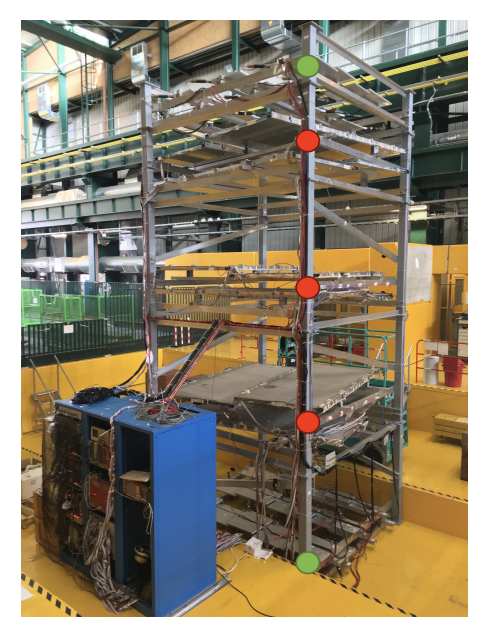

(b)

Figure 5: (a): schematic view of the MATHUSLA test stand. (b): picture of the final assembled structure in his test area in the ATLAS SX1 building at CERN. The green dots identify the two scintillator layers used for triggering, while the red dots the three RPC layers used for tracking.

\section{MATHUSLA as a cosmic ray telescope}

The design of MATHUSLA is driven by the requirements of reconstructing upward-traveling displaced vertices and distinguishing them from downward-traveling cosmic rays. Nevertheless, MATHUSLA has all the qualities needed to act as an excellent cosmic ray telescope [20]. The combination of a large area detector of atmospheric showers, that can measure both electron and muon particles, with a LHC detector, that can measure only muons, provides a more complete 
picture of cosmic air showers. In this framework, MATHUSLA can allow more detailed studies of the core structure, crucial to determine the atomic number of the primary cosmic particles. The study of cosmic rays is therefore an important secondary physics goal of MATHUSLA. These measurements, which do not interfere with the primary goal of LLP discovery, represent a "guaranteed physics return" on the investment of the detector, as well as an opportunity to establish a cosmic ray physics program.

\section{References}

[1] G.F. Giudice, A. Romanino, Split Supersymmetry, Nucl. Phys. B 699 (2004) 65-89, 10.1016/j.nuclphysb.2004.11.048, [arXiv: hep-ph/ 0406088 ].

[2] G. F. Giudice, R. Rattazzi, Theories with Gauge-Mediated Supersymmetry Breaking, Phys. Rept. 322 (1999) 419-499, 10.1016/S0370-1573(99)00042-3, [arXiv: hep-ph/9801271].

[3] R. Barbier et al., R-parity violating supersymmetry, Phys. Rept. 420 (2005) 1-202, 10.1016/j.physrep.2005.08.006, [arXiv: hep-ph/0 406039 ].

[4] J. Fan, M. Reece, J. T. Ruderman, Stealth Supersymmetry, JHEP 11 (2011) 12, 10.1007/JHEP11(2011)012, [arXiv:1105.5135 [hep-ph]].

[5] D. Curtin, C. B. Verhaaren, Discovering Uncolored Naturalness in Exotic Higgs Decays, JHEP 12 (2015) 72, 10.1007/JHEP12(2015)072, [arXiv:1506.06141 [hep-ph]].

[6] M. J. Strassler, K. M. Zurek, Echoes of a Hidden Valley at Hadron Colliders, Phys. Lett. B 651 (2007) 374-379, 10.1016/j.physletb.2007.06.055, [arXiv: hep-ph/ 0604261 ].

[7] M. J. Strassler, Possible Effects of a Hidden Valley on Supersymmetric Phenomenology, [arXiv:hep-ph/0607160].

[8] D. Tucker-Smith, N. Weiner, Inelastic dark matter, Phys. Rev. D 64 (2001) 043502, 10.1103/PhysRevD.64.043502, [arXiv: hep-ph/0101138].

[9] ATLAS Collaboration, Search for long-lived, weakly-interacting particles that decay to displaced hadronic jets in proton-proton collisions at $\sqrt{s}=8 \mathrm{TeV}$ with the ATLAS detector, Phys. Rev. D 92 (2015) 012010, 10.1103/PhysRevD.92.012010, [arXiv:1504.03634 [hep-ex]].

[10] ATLAS Collaboration, Search for heavy long-lived multi-charged particles in pp collisions at $\sqrt{s}=8$ TeV using the ATLAS detector, Eur. Phys. J. C 75 (2015) 362, 10.1140/epjc/s10052-015-3534-2, [arXiv:1504.04188 [hep-ex]].

[11] J. P. Chou, D. Curtin, H. J. Lubatti, New detectors to explore the lifetime frontier, Phys. Lett. B, 767 (2017) 29-36, 10.1016/j.physletb.2017.01.043, [arXiv:1606.06298 [hep-ph] ].

[12] D. Curtin et al., Long-Lived Particles at the Energy Frontier: The MATHUSLA Physics Case, arXiv:1806.07396 [hep-ph].

[13] CMS Collaboration, Searches for invisible decays of the Higgs boson in pp collisions at $\sqrt{s}=7,8$, and $13 \mathrm{TeV}$, JHEP 02 (2017) 135, 10.1007/JHEP02(2017)135, [arXiv: 1610.09218 [hep-ex] ].

[14] A. Coccaro, D. Curtin, H. J. Lubatti, H. Russell, J. Shelton, Data-driven Model-independent Searches for Long-lived Particles at the LHC, Phys. Rev. D 94113003 (2016), [arXiv: 1605.02742 [hep-ph].

[15] C. Csaki, M. Geller, O. Telem, A. Weiler, The Flavor of the Composite Twin Higgs, JHEP 09 (2016) 146, 10.1007/JHEP09(2016)146, [arXiv:1512.03427 [hep-ph]]. 
[16] CMS Collaboration, CMS physics: Technical design report, CERN-LHCC-2006-001, CMS-TDR-008-1.

[17] D. A. Timashkov, A. A. Petrukhin, New results on muon inelastic cross section and energy loss in rock, Proceedings 29th ICRC Pune 2005 Vol. 9 p. 89, [arXiv: hep-ph/0611008].

[18] Protvino et al., Technical Design Report for the Do forward trigger scintillator counters, D0-3237 (1997).

[19] Argo-YBJ, Layout and performance of RPCs used in the Argo-YBJ experiment, Nuclear Instruments and Methods in Physics Research Section A: Accelerators, Spectrometers, Detectors and Associated Equipment, 562 (2006) 92-96.

[20] M. A. Subieta Vasquez et al., MATHUSLA cosmic ray whitepaper, In preparation. 